\title{
Cost-effectiveness of a 12-month fixed-duration venetoclax treatment in combination with obinutuzumab in first-line, unfit chronic lymphocytic leukemia in the United States
}

\author{
Anuja Chatterjee, PhD; Sheila Shapouri, PharmD, MS; Beenish S Manzoor, PhD, MPH; Arliene Ravelo, MPH; \\ Kavita Sail, PhD; Venetia Qendri, PhD; Gijs van de Wetering, PhD; and Matthew S Davids, MD, MMSc
}

\section{What is already known about this subject}

- Chronic lymphocytic leukemia (CLL) represents a substantial health and economic burden, with a 5-year survival of approximately $87 \%$ and a mean health care cost of $\$ 1,885$ per patient per month (for CLL-related events) in the United States.

- Current standard of care for patients over 65 years of age with preexisting conditions is chemoimmunotherapy with bendamustine plus rituximab (BR) or chlorambucil plus obinutuzumab (GClb); other treatment options include chemotherapy-free regimens based on ibrutinib (Ibr) or acalabrutinib.

- Fixed-duration treatment, such as that offered by venetoclax plus obinutuzumab (VenG), has the potential to provide cost savings in CLL treatment when compared with chemotherapy-free regimens, which require continuous treatment until disease progression and thus impose a substantial burden.

\section{What this study adds}

- This cost-effectiveness model shows that, for first-line unfit patients with CLL, VenG as a 12-month fixed-duration treatment offers lower projected costs and more quality-adjusted life-years (QALYs) gained when compared with other treat-to-progression regimens (ie, GClb, BR, Ibr, Ibr+R, and Ibr+G).

- At a threshold of $\$ 150,000$ per QALY gained, VenG provides value for money to US health care payers.

\section{ABSTRACT}

BACKGROUND: Chronic lymphocytic leukemia (CLL) is a significant health and economic burden in the United States. Treatments include chemoimmunotherapy, such as obinutuzumab (G) plus chlorambucil (Clb) or bendamustine plus rituximab (BR), and chemotherapy-free regimens incorporating oral targeted therapies such as ibrutinib (Ibr),

\author{
Author affiliations \\ Anuja Chatterjee, PhD, Pharmerit - An OPEN \\ Health Company, York, United Kingdom. \\ Sheila Shapouri, PharmD, MS, and Arliene \\ Ravelo, MPH, Genentech Inc., South San \\ Francisco, CA. Beenish S Manzoor, PhD, \\ $\mathrm{MPH}$, and Kavita Sail, PhD, AbbVie Inc., \\ North Chicago, IL. Venetia Qendri, PhD, and \\ Gijs van de Wetering, PhD, Pharmerit - An \\ OPEN Health Company, Rotterdam, The \\ Netherlands. Matthew S Davids, MD, MMSc, \\ Department of Medical Oncology, Dana- \\ Farber Cancer Institute, Boston, MA. \\ AUTHOR CORRESPONDENCE: \\ Anuja Chatterjee, +44 (0)1904 211471; \\ AnujaChatterjee@openhealthgroup.com
}

J Manag Care Spec Pharm. 2021;27(11):1532-44

Copyright $(2021$, Academy of Managed Care Pharmacy. All rights reserved.

acalabrutinib (Acala), or venetoclax (Ven). Most chemotherapy-free regimens require continuous treatment to progression, while Ven plus $G(V e n G)$ is given for a fixed duration of 12 months, based on the CLL14 trial 
that led to its approval. Fixed-duration VenG has the potential for cost savings compared with treat-to-progression chemotherapy-free regimens.

OBJECTIVE: To evaluate the cost-effectiveness of 12 months fixedduration VenG in first-line treatment of unfit patients with CLL from a US health care payer perspective compared with $\mathrm{GClb}, \mathrm{BR}, \mathrm{Ibr}, \mathrm{Ibr}+\mathrm{G}$, $\mathrm{lbr}+\mathrm{R}$, Acala, and Acala $+\mathrm{G}$.

METHODS: A partitioned survival model was developed with 3 health states: progression-free survival (PFS), postprogression survival, and dead. The patient population, as based on the CLL14 trial, comprised previously untreated unfit patients with CLL (mean age 71.1 years, $33.1 \%$ female). The distribution of patients in each health state over time was estimated using extrapolated PFS and overall survival (OS) curves for VenG and GClb, based on CLL14 data 2 or more years after treatment cessation. PFS and OS for the other comparators were estimated using hazard ratios vs VenG, based on a network metaanalysis. Adverse events, utility values, and costs were obtained from published literature. The model estimated life-years gained, qualityadjusted life-years (QALYs) gained, and costs. The time horizon was 20 years, with a cycle time of 28 days. Outcomes and costs were discounted at $3.0 \%$ per year, and costs were estimated from a US health care payer perspective. One-way and probabilistic sensitivity analyses were conducted.

RESULTS: In this cross-trial analysis of unfit CLL patients, in the base case, VenG had lower projected total costs than all comparators investigated. VenG also had larger projected health benefits (more QALYs gained) than GClb, BR, Ibr, and Ibr + R. VenG was therefore more effective and less costly than these comparators (dominant). $\mathrm{Ibr}+\mathrm{G}$, Acala, and Acala $+\mathrm{G}$ showed higher QALYs gained vs VenG $(0.022,0.672$, and 0.961 , respectively), and substantially higher projected costs vs VenG $(\$ 1,488,400, \$ 1,579,737$, and $\$ 1,656,154$, respectively). Thus, Ibr $+G$, Acala, and $A c a l a+G$ would cost more than $\$ 1,000,000$ per QALY gained vs VenG. At the commonly used willingness-to-pay threshold of $\$ 150,000$ per QALY gained, Ibr $+\mathrm{G}$, Acala, and Acala $+G$ were not cost-effective compared with VenG.

CONCLUSIONS: Fixed-duration VenG for 12 months is a cost-effective first-line treatment option for unfit CLL patients compared with other available options and provides value for money to US health care payers at a threshold of $\$ 150,000$ per QALY gained. Future studies with longer trial follow-up and more mature survival data may help to confirm longer-term cost benefits of VenG.

Chronic lymphocytic leukemia (CLL) represents a significant health burden in the United States, with over 186,000 people estimated to be living with the disease in 2017. ${ }^{1}$ Data from the Surveillance, Epidemiology, and End Results Program estimated 21,040 new cases of CLL and 4,060 CLL-related deaths in the United States in $2020 .^{2}$ In recent years, 5-year survival has improved from $79.0 \%$ for cases diagnosed in
2000 to $86.5 \%$ for cases diagnosed in $2012 .{ }^{1}$ The economic burden of CLL is also substantial. In a US retrospective study conducted from 2012 to 2015 using a representative sample of 7,639 patients with a CLL diagnosis and with private health insurance, the mean health care cost was $\$ 3,784$ per patient per month for all causes and $\$ 1,885$ per patient per month for CLL-related events. ${ }^{3}$

First-line CLL treatment options depend on the disease state, genetic abnormalities, the patient's age and the presence of coexisting conditions. ${ }^{4}$ Fludarabine, cyclophosphamide, and rituximab were established as first-line treatment in physically fit patients with CLL based on a trial in Germany. ${ }^{5}$ For patients aged over 65 years or with preexisting conditions, chlorambucil plus obinutuzumab (GClb) has been an established standard of care based on the CLL11 trial. ${ }^{6,7}$ Other chemoimmunotherapy regimens in CLL include bendamustine plus rituximab (BR). ${ }^{8}$

More recently, oral targeted therapy using small-molecule inhibitors of Bruton tyrosine kinase (BTK), such as ibrutinib (Ibr) and acalabrutinib (Acala), has been introduced for first-line treatment of patients with CLL. ${ }^{9}$ Venetoclax is another orally available small molecule that promotes apoptosis in CLL cells by mimicking physiological antagonists of the pro-survival protein B-cell-lymphoma-2, hence facilitating CLL cell death..$^{10,11}$ In a recent report from the prospective informCLL registry of US patients with CLL who were enrolled from 2015 to 2018, the most common first-line treatments (received by $\geq 20 \%$ of patients) were Ibr (as monotherapy or in combination, 39\%) and chemoimmunotherapy with BR (20\%); the most common treatment in the relapsed/refractory setting was Ibr (as monotherapy or in combination, $51 \%)^{12}$

The treatment options available for CLL have different implications for costs involved in disease management. Chemotherapy-free regimens based on BTK inhibitors such as Ibr or Acala, ${ }^{13-17}$ or idelalisib, ${ }^{9}$ require continuous treatment until disease progression or significant toxicity. In contrast, chemoimmunotherapy regimens are administered for a finite duration, and a complete course of treatment typically costs less than a single year of Ibr or idelalisib. ${ }^{9}$ In the United States, a modeling exercise projected that the per-patient lifetime cost of CLL treatment would increase by over $300 \%$ from 2011 to 2025 as oral targeted therapies become first-line treatment. ${ }^{9}$ Thus, although oral targeted therapies offer improved health benefits and survival, their high costs also impose a substantial financial burden on patients and payers. ${ }^{9}$

Venetoclax plus the anti-CD20 monoclonal antibody obinutuzumab/GA101 (VenG) is a chemotherapy-free combination approved by the US Food and Drug Administration in May 2019 for first-line treatment of CLL in adults. ${ }^{18}$ 
In the phase 3 CLL14 trial, VenG demonstrated longer progression-free survival (PFS) compared with GClb, the established standard of care, in treatment-naive patients with CLL and coexisting conditions (unfit patients). ${ }^{6}$ This clinical benefit was maintained over time; PFS was still significantly higher with VenG than GClb 2 years after the end of treatment..$^{19}$

VenG treatment is given for a fixed duration of 12 months and is currently the only fixed-duration chemotherapy-free treatment for untreated CLL in patients with coexisting conditions. ${ }^{6,20}$ VenG has the potential to offer cost savings in CLL treatment compared with chemotherapy-free regimens based on BTK inhibitors, which require continuous treatment until progression. ${ }^{21}$ Health care payers will need a framework to assess the relative costs and costeffectiveness of alternative treatment options, in order to make informed decisions on treatments for patients with CLL, using the latest data on clinical efficacy and cost.

The purpose of this study was to evaluate the costeffectiveness of VenG in first-line treatment of CLL from a US health care payer perspective compared with a range of other first-line CLL treatment options, using clinical data from the CLL14 trial.

\section{Methods}

\section{MODEL DESIGN}

A partitioned survival model was developed from a US health care system perspective to compare the cost-effectiveness of VenG with that of other first-line CLL treatment options over a lifetime horizon (20 years). The model included 3 health states: PFS, postprogression survival, and dead. A partitioned survival model structure was selected in preference to other model structures (Markov, semi-Markov, and discrete event simulation) because it accommodates the immature overall survival (OS) data available from the CLL14 trial and can ensure that the clinical trial data are followed closely. Furthermore, a partitioned survival model was preferred over a Markov model, which would require individual calculation of transition probabilities, thereby introducing further uncertainty into the results.

The model was constructed in alignment with decision modeling guidelines from the International Society for Pharmacoeconomics and Outcomes Research and the National Institute for Health and Care Excellence (NICE) guide to technology appraisal. ${ }^{22,23}$ The chosen model structure and associated assumptions were reviewed by the NICE appraisal committee and Evidence Review Group and were considered to be appropriate for the decision problem. ${ }^{24}$
The patient population distributions within each health state over time were estimated using extrapolated PFS and OS curves. The cycle length was 28 days. Half-cycle correction was applied to approximate costs and quality-adjusted life-years (QALYs) in every cycle. Outcomes and costs were discounted at $3.0 \%$ per year. ${ }^{25}$ Indirect costs such as costs attributed to losses in productivity were not considered.

\section{POPULATION MODELED}

The population from the CLL14 clinical trial (NCT02242942), composed of previously untreated unfit patients with CLL with a Cumulative Illness Rating Scale score of more than 6 , was modeled. Mean age was 71.1 years, and $33.1 \%$ of patients were female $(n=432){ }^{6,19}$ Body surface area was estimated from the height and weight of the CLL14 patient population at baseline using the Dubois formula. ${ }^{26}$

\section{INTERVENTION AND COMPARATORS}

Twelve-month fixed-duration treatment with VenG was compared with the following comparators: GClb, BR, Ibr, $\mathrm{Ibr}+\mathrm{R}, \mathrm{Ibr}+\mathrm{G}$, Acala monotherapy, and Acala $+\mathrm{G}$. US clinical guidelines were followed to guide the dosing regimens used in the model, which are summarized in Supplementary Table 1 (available in online article).

\section{MODEL OUTCOMES}

The model estimated life-years (LYs) gained, based on extrapolated PFS and OS, QALYs gained, and costs. QALYs were calculated by multiplying the utility for each health state by the time spent in that health state, and the utility decrement for each adverse event (AE) was calculated by multiplying the disutility by the estimated duration of the AE. Costs comprised CLL treatment, routine care and monitoring, $\mathrm{AE}$ costs, disease progression costs, and costs incurred due to end-of-life care. Incremental costs, incremental LYs, incremental QALYs, and the incremental cost-effectiveness ratio (ICER) were estimated for VenG vs each comparator.

\section{SURVIVAL CURVE ESTIMATION}

PFS and OS for VenG and GClb were extrapolated over the model time horizon based on the most recent results available from CLL14, with data collected at least 2 years after treatment cessation (August 2019 data cut). ${ }^{19}$ PFS was extrapolated using independent model fitting based on a log-logistic distribution. OS was extrapolated using dependent model fitting and an exponential distribution.

Models were assessed using goodness-of-fit based on model fit statistics (Akaike Information Criterion [AIC] and Bayesian Information Criterion [BIC]); visual fit following the recommendations in the NICE technical support 
document 1427; hazard pattern; landmark survival from external data; and expert opinion on landmark survival. The curves selected were compared with external evidence from a recent trial in a comparable patient population (CLL11) and validated by clinical experts in the field of CLL. ${ }^{7}$ The PFS and OS curves used are presented in Supplementary Figure 1 and Supplementary Figure 2, respectively (both available in online article).

To account for death due to other causes, the OS and PFS estimates from the original source were corrected for background mortality under the assumption that the ageand sex-adjusted mortality risk of patients with CLL can never be lower than the age- and sex-adjusted mortality risk of the general population. Background mortality was obtained from US life tables and age- and sex-adjusted to match the modeled patient population. ${ }^{28}$

In the base case, it was assumed based on expert opinion that there was no difference in OS between VenG and GClb, since patients would be salvaged quite quickly after first relapse. They would receive similar treatment options, and the clinical efficacy of the subsequent treatment line would be comparable. The experts were also shown the OS curves and the landmark survival results from the extrapolations. Based on these, they agreed that they would not expect to see a treatment effect on OS outcomes (a conservative option).

PFS and OS for comparators other than GClb were estimated using hazard ratios (HRs) vs VenG. These were based on a network meta-analysis (NMA) of trials identified following a systematic literature review, which was presented at the European Hematology Association Annual Congress in 2020. ${ }^{29}$ For the present study, the NMA was updated with 2 more comparators, Acala and Acala $+\mathrm{G}^{15}$ The trials in the NMA are summarized in Supplementary Figure 3, and the HRs for each comparator are shown in Supplementary Table 2 (both available in online article).

\section{MODEL PARAMETERS: ADVERSE EVENTS}

The probability of AEs for VenG and GClb was obtained from CLL14 trial data. For the other comparators, AE probabilities were obtained from published sources identified from the systematic review. ${ }^{8,15,30-32}$ The model inputs are summarized in $\underline{\text { Supplementary Table } 3}$ (available in online article).

\section{MODEL PARAMETERS: HEALTH STATE UTILITIES}

According to clinical experts, the utility estimates derived from the CLL14 trial were too high for the first-line CLL population; therefore, utility data for each health state were obtained from a systematic literature review and disutility data for AEs from other published literature. ${ }^{33-40}$ Utility data inputs are summarized in Table 1.

\section{MODEL PARAMETERS: COST INPUTS}

Cost inputs are summarized in Table 1. The drug costs for each comparator were based on dosing regimens from US prescribing information and current drug acquisition costs. Active treatment costs for VenG and GClb were based on time on treatment in the CLL14 trial (12 cycles venetoclax or chlorambucil plus 6 cycles obinutuzumab; Supplementary Table 1). The other comparators included fixed-duration treatment and treat-to-progression regimens. PFS curves for the comparators were estimated using the HR data from the NMA and were used to inform the number of patients on treatment in each cycle.

After disease progression, the subsequent treatment mix for patients initially treated with each of the comparators was based on US clinical expert advice. For patients initially treated with VenG, the subsequent treatment mix was Ibr in $50 \%$ of patients, Ven $+\mathrm{R}$ in $25 \%$, and Ven monotherapy in $25 \%$. For patients treated with chemoimmunotherapy regimens, the subsequent treatment mix was assumed to be the same as in the GClb arm of the CLL14 trial: Ibr in $75 \%$ of patients, Ven $+\mathrm{R}$ in $20 \%$, and Ven monotherapy in $5 \%$. The subsequent treatment mix in patients treated with Acala and Acala $+\mathrm{G}$ was assumed to be the same as in those treated with $\mathrm{Ibr}, \mathrm{Ibr}+\mathrm{R}$, and $\mathrm{Ibr}+\mathrm{G}$ : Ven $+\mathrm{R}$ in $25 \%$ of patients and Ven monotherapy in $75 \%$.

Time to next treatment (TTNT) for VenG and GClb was extrapolated from CLL14 trial data by independent model fitting using a log-logistic distribution. For the other comparators, TTNT was based on the difference between treatment-specific OS and PFS extrapolated curves. The duration of subsequent treatment was based on published literature and validated by a clinical expert. ${ }^{41-43}$

Costs associated with AEs were obtained from published literature. ${ }^{4-47}$ Cost of terminal care was applied as a one-off cost for all patients entering the death state in the model and was estimated at $\$ 19,695$, based on a published estimate from a study of patients with CLL, ${ }^{48}$ inflated to 2019 costs using US Bureau of Labor statistics data.$^{49}$ Monitoring costs were obtained from Centers for Medicare \& Medicaid Services published fee schedules and published literature. ${ }^{50}$

\section{SENSITIVITY ANALYSIS}

A one-way sensitivity analysis was conducted by varying key model parameters, including time on subsequent treatment, AE parameters, costs, utility values, HR, age, and percentage of females (Supplementary Table 4, available in online article). The impact of survival parameters was not tested in the one-way sensitivity analysis but was investigated in scenario analyses and included in the probabilistic sensitivity analysis (PSA). A PSA was conducted by simultaneously 


\section{TABLE 1 Key Input Data}

\begin{tabular}{|c|c|c|}
\hline Parameter & Base case value & Reference \\
\hline Preprogression health state utility (all treatments) & 0.670 & NICE TA34333 \\
\hline Postprogression health state utility (all treatments) & 0.600 & NICE TA34333 \\
\hline End-of-life costs & $\$ 19,695.00$ & $\begin{array}{l}\text { Lafeuille et al }{ }^{48} \text { (inflated to } 2019 \text { costs using Bureau of Labor } \\
\text { Statistics data) }{ }^{49}\end{array}$ \\
\hline \multicolumn{3}{|l|}{ Monitoring costs } \\
\hline Tumor lysis syndrome prophylaxis & $\$ 15,686.00$ & Cho et $\mathrm{al}^{53}$ \\
\hline Full blood count & $\$ 8.63$ & $\begin{array}{l}\text { CMS Clinical Diagnostic Laboratory Fee Schedule } 2019 \\
\text { (HCPCS } 85025 \text { complete blood count with automated } \\
\text { differential white blood cells) }\end{array}$ \\
\hline Lactate dehydrogenase & $\$ 6.71$ & $\begin{array}{l}\text { CMS Clinical Diagnostic Laboratory Fee Schedule } 2019 \\
\text { (HCPCS } 83615 \text { lactate dehydrogenase enzyme) }\end{array}$ \\
\hline Chest x-ray & $\$ 25.23$ & CMS Physician Fee Schedule 2019 (CPT 71045) \\
\hline Bone marrow examination & $\$ 72.08$ & CMS Physician Fee Schedule 2019 (CPT 38221) \\
\hline Hematologist visit & $\$ 51.90$ & $\begin{array}{l}\text { CPT } 99213 \text { - office/outpatient visit (same CPT as Chen } 2017^{9} \text { )- } \\
\text { CMS Physician Fee Schedule } 2019\end{array}$ \\
\hline Inpatient nonsurgical/medical visit & $\$ 690.10$ & $\begin{array}{l}\text { Wang et al. }{ }^{50} \text { Hospitalization costs. Inflated from } 2019 \text { to } 2019 \\
\text { using CPI }\end{array}$ \\
\hline Full blood transfusion & $\$ 494.08$ & $\begin{array}{l}\text { CMS OPPS Cost Statistics File 2019: Whole blood for } \\
\text { transfusion cost (HCPCS P9010) + CMS Physician Fee Schedule } \\
2019 \text { (CPT 36430) }\end{array}$ \\
\hline Lymphocyte count & $\$ 2.82$ & $\begin{array}{l}\text { CMS Clinical Diagnostic Laboratory Fee Schedule } 2019 \\
\text { (HCPCS } 85048 \text { automated leukocyte count) }\end{array}$ \\
\hline CT scan & $\$ 199.30$ & CMS Physician Fee Schedule 2019 (CPT 71260) \\
\hline Biochemistry test: renal - urea and electrolytes test & $\$ 11.74$ & $\begin{array}{l}\text { CMS Clinical Diagnostic Laboratory Fee Schedule } 2019 \\
\text { (HCPCS } 80053 \text { comprehensive metabolic panel) }\end{array}$ \\
\hline Biochemistry test: liver function test & $\$ 9.08$ & $\begin{array}{l}\text { CMS Clinical Diagnostic Laboratory Fee Schedule } 2019 \\
\text { (HCPCS } 80076 \text { hepatic function panel) }\end{array}$ \\
\hline Immunoglobulins blood test & $\$ 13.84$ & $\begin{array}{l}\text { CMS Clinical Diagnostic Laboratory Fee Schedule } 2019 \\
\text { (HCPCS } 86023 \text { comprehensive metabolic panel) }\end{array}$ \\
\hline Drug costs (pack size) & Cost per pack & \\
\hline Venetoclax (120×100 mg tablets) & $\$ 12,293.47$ & $\begin{array}{l}\text { NDC: } 00074-0579-28, \text { RED BOOK, last accessed } \\
\text { January } 28,2020\end{array}$ \\
\hline Obinutuzumab (25 mg/mL, $40 \mathrm{~mL})$ & $\$ 6,649.27$ & $\begin{array}{l}\text { NDC: } 00074-0579-28, \text { RED BOOK, last accessed } \\
\text { January } 28,2020\end{array}$ \\
\hline Chlorambucil ( $25 \times 2$ mg tablets) & $\$ 608.19$ & $\begin{array}{l}\text { NDC: } 00074-0579-28, \text { RED BOOK, last accessed } \\
\text { January } 28,2020\end{array}$ \\
\hline Rituximab IV (10 mg/mL, $10 \mathrm{~mL})$ & $\$ 939.52$ & $\begin{array}{l}\text { NDC: } 00074-0579-28, \text { RED BOOK, last accessed } \\
\text { January } 28,2020\end{array}$ \\
\hline Bendamustine IV (25 mg/mL, $4 \mathrm{~mL})$ & $\$ 2,473.80$ & $\begin{array}{l}\text { NDC: } 00074-0579-28, \text { RED BOOK, last accessed } \\
\text { January } 28,2020\end{array}$ \\
\hline Ibrutinib ( $28 \times 420 \mathrm{mg}$ tablets) & $\$ 12,966.10$ & $\begin{array}{l}\text { NDC: } 00074-0579-28, \text { RED BOOK, last accessed } \\
\text { January } 28,2020\end{array}$ \\
\hline Acalabrutinib (60×100 mg tablets) & $\$ 14,064.00$ & $\begin{array}{l}\text { NDC: } 00074-0579-28, \text { RED BOOK, last accessed } \\
\text { January } 28,2020\end{array}$ \\
\hline
\end{tabular}


TABLE 1 Key Input Data (continued)

\begin{tabular}{|c|c|c|}
\hline Parameter & Base case value & Reference \\
\hline \multicolumn{3}{|c|}{ Subsequent treatment drug acquisition cost } \\
\hline VenG & $\$ 104,477.63$ & \multirow{8}{*}{$\begin{array}{l}\text { Subsequent treatment costs determined by: } \\
\text { - Subsequent treatment mix, derived from US clinical } \\
\text { expert opinion (Ven+R, Ibr, and Ven monotherapy, relative } \\
\text { proportion of patients receiving each dependent upon initial } \\
\text { treatment). } \\
\text { - Time point for start of next treatment, derived from the } \\
\text { TTNT curves for the VenG and GClb arms from the CLL14 } \\
\text { trial. } \\
\text { - Time on treatment derived from published data: Ven }+R^{,}, 1,54 \\
\text { validated by clinical expert; Ibrutinib, }{ }^{42} \text { validated by clinical } \\
\text { expert; Ven monotherapy }\end{array}$} \\
\hline GClb & $\$ 467,032.63$ & \\
\hline $\mathrm{BR}$ & $\$ 467,032.63$ & \\
\hline $\mathrm{Ibr}$ & $\$ 211,212.97$ & \\
\hline $\mathrm{Ibr}+\mathrm{G}$ & $\$ 211,212.97$ & \\
\hline $\mathrm{Ibr}+\mathrm{R}$ & $\$ 211,212.97$ & \\
\hline Acala & $\$ 112,737.90$ & \\
\hline Acala $+\mathrm{G}$ & $\$ 25,861.77$ & \\
\hline \multicolumn{3}{|l|}{ AE costs } \\
\hline Asthenia & $\$ 4,810.18$ & Borker ${ }^{44}$ \\
\hline Diarrhea & $\$ 5,172.65$ & Barnes et al ${ }^{45}$ \\
\hline Dyspnea & $\$ 11,903.85$ & Wong et $\mathrm{al}^{47}$ \\
\hline Febrile neutropenia & $\$ 13,545.98$ & $\mathrm{AHRQ}^{46}$ \\
\hline Infusion-related reaction & $\$ 7,289.00$ & $\mathrm{AHRQ}^{46}$ \\
\hline Leukopenia & $\$ 6,911.00$ & $\mathrm{AHRQ}^{46}$ \\
\hline Neutropenia & $\$ 13,565.69$ & $\mathrm{AHRQ}^{46}$ \\
\hline Pneumonia & $\$ 9,046.00$ & $\mathrm{AHRQ}^{46}$ \\
\hline Sepsis & $\$ 17,661.00$ & $\mathrm{AHRQ}^{46}$ \\
\hline Thrombocytopenia & $\$ 10,017.00$ & $\mathrm{AHRQ}^{46}$ \\
\hline \multicolumn{3}{|l|}{ AE disutilities } \\
\hline Asthenia & 0.115 & NICE TA306 34 \\
\hline Diarrhea & 0.080 & NICE TA216 35 \\
\hline Dyspnea & 0.103 & NICE TA306 34 \\
\hline Febrile neutropenia & 0.150 & NICE TA34333 \\
\hline Infusion-related reaction & 0.200 & NICE TA34333 \\
\hline Leukopenia & 0.090 & Assumed same as neutropenia \\
\hline Neutropenia & 0.090 & $\begin{array}{l}\text { NICE TA } 343^{33} \\
\text { Nafees et } a^{36}\end{array}$ \\
\hline Pneumonia & 0.195 & Tolley et $\mathrm{al}^{37}$ \\
\hline Sepsis & 0.195 & Tolley et $\mathrm{al}^{37}$ \\
\hline Thrombocytopenia & 0.108 & Tolley et $\mathrm{al}^{37}$ \\
\hline
\end{tabular}

Acala=acalabrutinib; $A E=$ adverse event; $A H R Q=A g e n c y$ for Healthcare Research and Quality; $B=$ bendamustine; $C l b=$ chlorambucil; $C M S=C e n t e r s$ for Medicare \& Medicaid Services; $\mathrm{CPI}=$ Consumer Price Index; $C P T=C$ urrent Procedural Terminology; $C T=$ computed tomography; $G=0$ binutuzumab; $G C l b=0 b i n u t u z u m a b$ plus chlorambucil; HCPCS= Healthcare Common Procedure Coding System; Ibr=ibrutinib; IV=intravenous; NDC= National Drug Code; NICE = National Institute for Health and Care Excellence; $R=$ rituximab; TTNT = time to next treatment; Ven=venetoclax.

sampling model parameter values from assigned distributions for 1,000 iterations of the model.

Scenario analyses were performed using different discount rates, shorter time horizons of 5 years or 10 years, and different utility values. Another scenario analysis tested the effect of using alternative HRs adjusted for the heterogeneity in GClb dosing across trials (during the NMA feasibility assessment stage, it was observed that treatment duration 


\begin{tabular}{|c|c|c|c|c|c|c|c|}
\hline Treatment & $\begin{array}{l}\text { Total costs } \\
\text { (\$) }\end{array}$ & $\begin{array}{l}\text { Life-years } \\
\text { gained }\end{array}$ & $\begin{array}{l}\text { QALYs } \\
\text { gained }\end{array}$ & $\begin{array}{l}\text { Incremental } \\
\text { costs }(\$)\end{array}$ & $\begin{array}{c}\text { Incremental } \\
\text { life-years gained }\end{array}$ & $\begin{array}{l}\text { Incremental } \\
\text { QALYs gained }\end{array}$ & $\begin{array}{c}\text { ICER } \\
\text { (\$/QALY) }\end{array}$ \\
\hline VenG & $\$ 291,012$ & 13.01 & 6.521 & - & - & - & - \\
\hline GClb & $\$ 491,040$ & 13.01 & 6.188 & $\$ 200,028$ & 0 & -0.333 & VenG is dominant \\
\hline$B R$ & $\$ 595,771$ & 12.31 & 5.815 & $\$ 304,759$ & -0.70 & -0.706 & VenG is dominant \\
\hline $\mathrm{Ibr}$ & $\$ 1,045,472$ & 12.31 & 6.004 & $\$ 754,460$ & -0.70 & -0.517 & VenG is dominant \\
\hline $\mathrm{Ibr}+\mathrm{G}$ & $\$ 1,779,412$ & 13.02 & 6.543 & $\$ 1,488,400$ & 0.01 & 0.022 & $\$ 67,856,575$ \\
\hline $\mathrm{Ibr}+\mathrm{R}$ & $\$ 1,040,860$ & 12.22 & 5.946 & $\$ 749,848$ & -0.79 & -0.576 & VenG is dominant \\
\hline Acala & $\$ 1,870,749$ & 13.55 & 7.194 & $\$ 1,579,737$ & 0.54 & 0.672 & $\$ 2,349,304$ \\
\hline Acala + G & $\$ 1,947,166$ & 13.56 & 7.482 & $\$ 1,656,154$ & 0.55 & 0.961 & $\$ 1,724,052$ \\
\hline
\end{tabular}

for Clb varied across trials). For example, in the CLL14 trial, patients received up to 14 cycles of GClb, whereas in the CLL11 study, the GClb arm had a treatment duration of only 6 cycles. The Clb-adjusted HRs were not used in the base-case option, primarily because these would introduce further uncertainty into the model outcomes. In addition, adjusting for dosing would likely favor VenG; therefore, we proceeded with a conservative base case assumption.

Two further scenario analyses tested the effect of using alternative parametric curves, one assuming a treatment effect for OS using a dependent model and exponential distribution, and one assuming no treatment effect for OS using a dependent model and log-normal distribution. The base-case scenario assumed no treatment effect between VenG and GClb. The scenario under which a dependent model with a log-normal distribution was used to model OS was supported by the next best distribution fit based on AIC and BIC criteria.

\section{Results}

\section{BASE-CASE RESULTS}

Table 2 summarizes the base-case cost-effectiveness results for each comparator. VenG had lower projected total costs $(\$ 291,012)$ than all comparators investigated (highest projected total costs, \$1,947,166 [Acala+G]). VenG also had larger projected health benefits (more QALYs gained [6.521]) than 4 of the comparators (GClb [6.188], BR [5.815], Ibr [6.004], and Ibr+R [5.946]). Thus, VenG was more effective and less costly than 4 of the comparators: GClb, BR, Ibr, and Ibr $+\mathrm{R}$. This position, where a treatment is more effective and less costly than an alternative, is referred to as the "dominant" treatment option.
The other 3 comparators, Ibr $+\mathrm{G}$, Acala, and Acala $+\mathrm{G}$, had higher projected health gains (more QALYs gained) than VenG in the base case $(6.543,7.194$, and 7.482 , respectively) and were also substantially more costly. Since the extra costs for Ibr $+\mathrm{G}$, Acala, and Acala $+\mathrm{G}$ compared with VenG were large (\$1,488,400, \$1,579,737, and \$1,656,154, respectively) and the extra QALY gains were small (0.022, 0.672, and 0.961 QALYs gained, respectively), these comparators would cost over $\$ 1,000,000$ per QALY gained compared with VenG. Therefore, at a willingness-to-pay threshold of $\$ 150,000$ per QALY, Ibr $+\mathrm{G}$, Acala, and Acala $+\mathrm{G}$ were not cost-effective vs VenG.

\section{ONE-WAY SENSITIVITY ANALYSIS}

The one-way sensitivity analysis for VenG vs GClb showed that the parameters with the largest impact on the ICER (incremental cost per QALY) were the preprogression and postprogression utility values (Figure 1). The strongest driver of the ICER was the postprogression survival utility value. Since a larger proportion of patients in the GClb arm remained in the postprogression survival period than in the VenG arm, the QALYs accrued in this health state had the largest impact on the ICER. Increasing the postprogression utility value resulted in higher QALYs gained for GClb than for VenG. Therefore, under this scenario, VenG was not the dominant treatment option. The other parameters tested had little impact on the ICER. The one-way sensitivity analysis did not include survival parameters, which were explored in scenario analyses and the PSA.

Results of the one-way sensitivity analysis for VenG vs the other comparators are shown in Supplementary Figures 4-9 (available in online article). For VenG vs BR, Ibr, Ibr + G, 


\section{FIGURE 1 Results of One-Way Deterministic Sensitivity Analysis, VenG vs GClb}

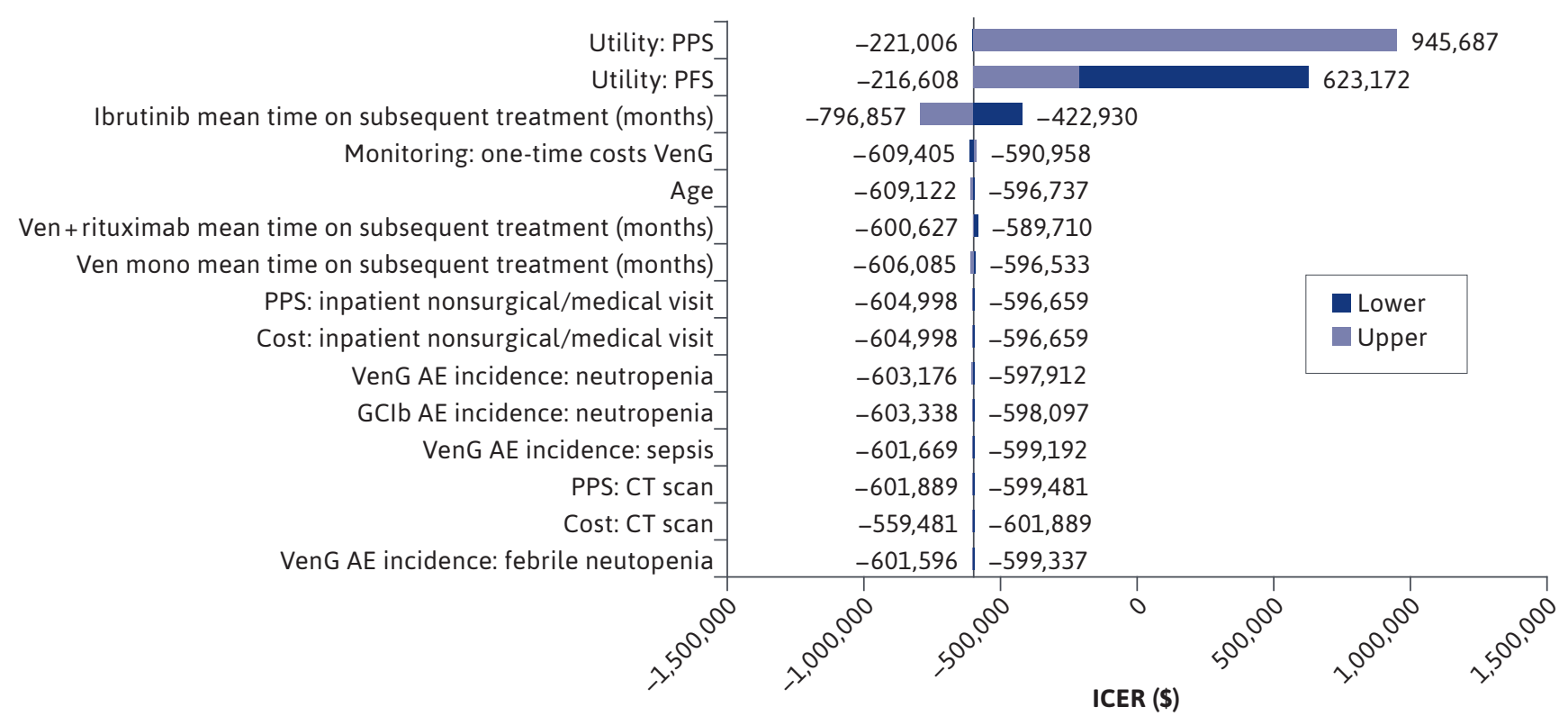

$A E=$ adverse event; $C l b=$ chlorambucil; $C T=$ computed tomography; $G=$ obinutuzumab; ICER =incremental cost-effectiveness ratio; mono=monotherapy; $P F S=$ progression-free survival; PPS = postprogression survival; Ven = venetoclax.

and $\mathrm{Ibr}+\mathrm{R}$, the postprogression and PFS utilities, followed by the OS HR, had the largest impact on the ICER. For VenG vs Acala or Acala $+G$, the PFS HR had the largest impact on the ICER. For Acala, the greatest driver of actual incremental costs was the PFS HR of Acala vs VenG, since the HR had a large impact on the PFS curve and, subsequently, the preprogression costs.

The PFS HR of Acala vs VenG also had the largest impact on incremental QALYs, since this parameter was a key driver of the survival curves for VenG and Acala, which in turn determined the incremental preprogression QALYs. For Acala $+\mathrm{G}$, the greatest impact on incremental costs was the OS HR of Acala $+G$ vs VenG, explained by the impact that the HR had on the OS curve, which in turn was a key determinant of incremental costs. The PFS HR of Acala $+\mathrm{G}$ vs VenG had the largest impact on incremental QALYs.

\section{PROBABILISTIC SENSITIVITY ANALYSIS}

Figure 2 shows the results of the PSA plotted on the costeffectiveness plane. At a willingness-to-pay threshold of \$150,000 per QALY, the probability of VenG being the most cost-effective treatment was over 90\% (Supplementary Figure 10, available in online article).

\section{SCENARIO ANALYSIS}

Supplementary Table 5 presents the total costs, LYs gained, and QALYs gained for each treatment, and the incremental costs, incremental LYs, incremental QALYs, and ICERs for each comparator vs VenG, in a scenario using alternative HRs adjusted for GClb dosing heterogeneity across trials. Under this scenario, VenG had lower projected total costs (\$291,012) and higher projected QALYs gained (6.521) than all other comparators (highest projected total cost, \$1,584,434 [Acala]; lowest projected QALYs gained, 3.365 [BR]).

Supplementary Tables 6 and 7 (available in online article) present the total costs, LYs gained, and QALYs gained for each treatment, and the incremental costs, incremental LYs, incremental QALYs, and ICERs for each comparator vs VenG, in 2 scenarios with alternative parametric curves. Supplementary Table 6 shows the effect of assuming a treatment effect for OS using a dependent model and exponential distribution, and Supplementary Table 7 shows the effect of assuming no treatment effect for OS using a dependent model and log-normal distribution.

The results of both these scenarios did not alter conclusions based on the base-case analysis. VenG was more effective and less costly than 4 of the comparators: GClb, BR, Ibr, and Ibr $+\mathrm{R}$. The other 3 comparators, Ibr $+\mathrm{G}$, Acala, and 


\section{FIGURE 2 Results of Probabilistic Sensitivity Analysis}

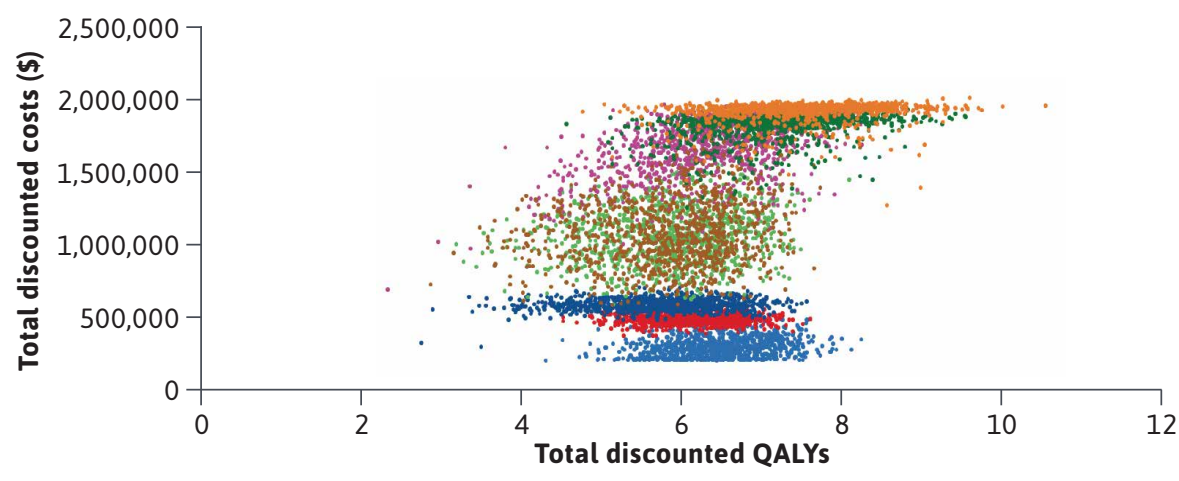

\begin{tabular}{|llll|}
\hline Venetoclax $+\mathrm{G}$ & Chlorambucil $+\mathrm{G}$ & Bendamustine $+\mathrm{G}$ & $\mathbf{\text { Ibruntinib }}$ \\
Ibruntinib $+\mathrm{G}$ & Ibruntinib $+\mathrm{R}$ & Acalabrutinib $+\mathrm{G}$ & Acalabrutinib $+\mathrm{G}$ \\
\hline
\end{tabular}

$G=$ obinutuzumab; $Q A L Y=$ quality-adjusted life-year; $R=$ rituximab

Acala $+\mathrm{G}$, had more projected QALYs gained than VenG and substantially higher projected costs than VenG. As in the base case, Ibr+G, Acala, and Acala $+\mathrm{G}$ were not cost-effective vs VenG at a willingness-to-pay threshold of $\$ 150,000$.

Additional scenario analyses tested different discount rates, shorter time horizons of 5 years or 10 years, and different utility values, with no significant impact on incremental QALYs. VenG remained the dominant treatment option in most of these scenarios (data not shown).

\section{Discussion}

To our knowledge, this is the first cost-effectiveness study comparing VenG with other first-line CLL treatments in unfit patients with CLL in the United States. The results of the CLL14 trial demonstrated the clinical benefit of VenG in this patient population, with longer PFS than GClb, which was maintained 2 years after the end of treatment. ${ }^{6,19}$ The results of our model build on the clinical data from
CLL14 and indicate that VenG is also a cost-effective option compared with other current treatments in first-line CLL. Our results showed that VenG had lower projected costs than any of the other CLL treatment options investigated. For 4 of the comparators included in the analysis $(\mathrm{GClb}, \mathrm{BR}$, $\mathrm{Ibr}$, and $\mathrm{Ibr}+\mathrm{R}$ ), VenG also had higher projected health benefits (more QALYs gained). Therefore, compared with GClb, BR, Ibr, or Ibr+R, VenG would be both more effective and less costly.

The only comparators with higher health gains than VenG in the basecase analysis were $\mathrm{Ibr}+\mathrm{G}$, Acala, and Acala $+G$. However, the ICERs for these treatments compared with VenG were more than $\$ 1,000,000$ per QALY. The United States does not have a single uniformly agreed upon threshold for acceptable cost-effectiveness of a medical intervention. The use of multiple thresholds has been recommended to allow for variations in available resources and possible alternative uses for those resources. ${ }^{51}$

In the absence of explicit resource constraints, a threshold of $\$ 150,000$ per QALY has been proposed and is broadly considered to represent good value for the money. ${ }^{51,52}$ The ICERs for $\mathrm{Ibr}+\mathrm{G}$, Acala, and Acala $+\mathrm{G}$ compared with VenG were considerably above the $\$ 150,000$ per QALY willingnessto-pay threshold. Therefore, these 3 comparators would not be considered cost-effective compared with VenG at this threshold. In our model, at a willingness-to-pay threshold of $\$ 150,000$ per QALY, the fixed-duration treatment combination of VenG had a probability of being the most costeffective treatment of over $90 \%$.

The one-way sensitivity analysis indicated that the results were robust to changes in most parameters. When comparing VenG with GClb, the parameters with the largest impact on the ICER were utility values in the progression-free and postprogression health states. For VenG vs BR, Ibr, $\mathrm{Ibr}+\mathrm{G}$, and $\mathrm{Ibr}+\mathrm{R}$, the parameters tested had little impact on the ICER. For VenG vs Acala or Acala $+G$, the parameter with the largest impact on the ICER was the PFS HR. The one-way sensitivity analysis did not evaluate the effect of survival parameters. Nonetheless, a scenario analysis using alternative HRs adjusted for $\mathrm{GClb}$ dosing heterogeneity across trials showed that VenG had lower projected total costs and higher projected QALYs gained than all other comparators, making VenG the dominant treatment option under this scenario.

Furthermore, scenarios using alternative parametric survival curves showed that base-case results were robust to changes in these parameters. Further scenario analyses using different discount rates, shorter time horizons, and different utility values confirmed that VenG was the dominant treatment option in most of these scenarios.

Strengths of this analysis include use of up-to-date evidence for the effect of VenG and GClb on PFS from 
the CLL14 clinical trial 2 years after treatment cessation. ${ }^{19}$ The choice of survival curves was validated by comparing with external evidence from a recent trial in a comparable population and reviewed by clinical experts. Furthermore, the economic model was also validated by health economic experts and was accepted in health technology assessment submissions, such as the submission to NICE. ${ }^{24}$

\section{LIMITATIONS}

The model, although comprehensive, has some limitations. The HRs from our NMA are a key determinant of the external comparators' outcomes in the current analysis; however, other recently published NMAs have noted similar findings, ${ }^{53,54}$ that is, that Acala and Acala $+\mathrm{G}$ achieve better PFS outcomes than VenG, thereby supporting the findings of our NMA (although it should be noted that the NMAs are not strictly comparable, since our NMA included only studies with unfit, previously untreated CLL populations). Further, since it is currently unknown whether patients who achieve remission after VenG but subsequently relapse benefit from repeat treatment with VenG, the current model does not consider the costs of VenG retreatment.

Additionally, at the cut-off date used in this analysis, the data from the CLL14 trial were still immature, since the median OS and TTNT had not yet been reached, and median PFS had been reached in the $\mathrm{GClb}$ arm but not in the VenG arm, which may affect the results presented here. ${ }^{19}$ In particular, long-term OS extrapolations are uncertain, and there was no significant difference in OS between VenG and GClb at the primary analysis of the CLL14 trial. ${ }^{6}$ Median OS had not yet been reached in either patient group at follow-up 2 years after treatment cessation. ${ }^{19}$
Extrapolating the patterns observed in the CLL14 data resulted in projected OS estimates that were close to background mortality. This is plausible, since the median age for the CLL14 patient population in the trial was 72 years and most had medical comorbidities, ${ }^{6}$ so they had a significant likelihood of dying from other causes that would be captured by background mortality.

\section{Conclusions}

The results of this cost-effectiveness model indicate that VenG, as a 12-month fixed-duration treatment, is a more cost-effective treatment option (when compared with other available options, such as chemoimmunotherapy and BTK inhibitor regimens) for first-line unfit patients with CLL and provides good value for money to US health care payers at a threshold of $\$ 150,000$ per QALY. Future analyses with longer follow-up are needed to confirm the continued cost benefit of VenG.

\section{DISCLOSURES}

Genentech Inc. and AbbVie provided financial support for this study. Genentech Inc., AbbVie, and Pharmerit-An OPEN Health Company participated in the design, study conduct, analysis, and interpretation of data, as well as the writing, review, and approval of the manuscript. Venetoclax is being developed in a collaboration between Genentech Inc. and AbbVie. Ravelo and Shapouri are employed by Genentech Inc. and have ownership interests. Manzoor and Sail are employed by AbbVie and have ownership interests. Chatterjee, van de Wetering, and Qendri, employees of Pharmerit - An OPEN Health Company, received consultancy fees from AbbVie.
Davids has received consultancy fees from AbbVie, AstraZeneca, Eli Lilly, Genentech Inc., Janssen, MEI Pharma, Novartis, Pharmacyclics, and Verastem; research funding from Ascentage Pharma, Genentech Inc., MEI Pharma, Pharmacyclics, Surface Oncology, TG Therapeutics, and Verastem; and has served on board of directors or advisory committees for AbbVie, Adaptive Biotechnologies, AstraZeneca, BeiGene, Eli Lilly, Genentech Inc., Janssen, Pharmacyclics, TG Therapeutics, and Verastem.

This study was presented as a poster at ASH 61st Annual Meeting and Exposition; December 7-10, 2019; Orlando, FL.

\section{ACKNOWLEDGMENTS}

Third-party medical writing assistance, under the direction of the authors, was provided by Simon Lancaster, BSc, and Carole Nadin, MPhil, contract medical writers for Ashfield MedComms, an Ashfield Health company, funded by F. Hoffmann-La Roche Ltd.

\section{DATA-SHARING STATEMENT}

Qualified researchers may request access to individual patient-level data through the clinical study data request platform (https://vivli.org/). Further details on Roche's criteria for eligible studies are available here: https://vivli.org/members/ourmembers/. For further details on Roche's Global Policy on the Sharing of Clinical Information and how to request access to related clinical study documents, see here: https://www.roche com/research_and_development/who_ we are how we work/clinical trials/ our_commitment_to_data_sharing.htm.

\section{REFERENCES}

1. Surveillance Epidemiology and End Results (SEER) Program. SEER Explorer. 2018. Accessed December 29, 2020. https://seer.cancer.gov/explorer/

2. Surveillance Epidemiology and End Results (SEER) Program. Table 1.1. Estimated new cancer cases and deaths for 2020. Accessed December 29, 2020. https://seer.cancer.gov/csr/1975_2017/ results single/sect 01 table.01.pdf 
3. Kabadi SM, Goyal RK, Nagar SP, Kaye JA, Davis KL. Treatment patterns, adverse events, and economic burden in a privately insured population of patients with chronic lymphocytic leukemia in the United States. Cancer Med. 2019;8(8):3803-10.

4. Eichhorst B, Robak T, Montserrat E, et al. Chronic lymphocytic leukaemia: ESMO Clinical Practice Guidelines for diagnosis, treatment and follow-up. Ann Oncol. 2015;26(Suppl 5):v78-84.

5. Hallek M, Fischer K, Fingerle-Rowson G, et al. Addition of rituximab to fludarabine and cyclophosphamide in patients with chronic lymphocytic leukaemia: a randomised, open-label, phase 3 trial. Lancet. 2010;376(9747):1164-74.

6. Fischer K, Al-Sawaf O, Bahlo J, et al. Venetoclax and obinutuzumab in patients with CLL and coexisting conditions. N Engl J Med. 2019;380(23):2225-36.

7. Goede V, Fischer K, Busch R, et al. Obinutuzumab plus chlorambucil in patients with CLL and coexisting conditions. N Engl J Med. 2014;370(12):1101-10.

8. Eichhorst B, Fink AM, Bahlo J, et al. First-line chemoimmunotherapy with bendamustine and rituximab versus fludarabine, cyclophosphamide, and rituximab in patients with advanced chronic lymphocytic leukaemia (CLL10): an international, open-label, randomised, phase 3, non-inferiority trial. Lancet Oncol. 2016;17(7):928-42.

9. Chen Q, Jain N, Ayer T, et al. Economic burden of chronic lymphocytic leukemia in the era of oral targeted therapies in the United States. J Clin Oncol. 2017;35(2):166-74.

10. Souers AJ, Leverson JD, Boghaert ER, et al. ABT-199, a potent and selective BCL-2 inhibitor, achieves antitumor activity while sparing platelets. Nat Med. 2013;19(2):202-08.

11. Roberts AW, Davids MS, Pagel JM, et al. Targeting BCL2 with venetoclax in relapsed chronic lymphocytic leukemia. N Engl J Med. 2016;374(4):311-22.
12. Mato AR, Barrientos JC, Ghosh N, et al. Prognostic testing and treatment patterns in chronic lymphocytic leukemia in the era of novel targeted therapies: results from the informCLL registry. Clin Lymphoma Myeloma Leuk. 2020;20(3): 174-83.e3

13. Awan FT, Schuh A, Brown JR, et al. Acalabrutinib monotherapy in patients with chronic lymphocytic leukemia who are intolerant to ibrutinib. Blood Adv. 2019;3(9):1553-62.

14. Byrd JC, Wierda WG, Schuh A, et al. Acalabrutinib monotherapy in patients with relapsed/refractory chronic lymphocytic leukemia: updated phase 2 results. Blood. 2020;135(15):1204-13.

15. Sharman JP, Egyed M, Jurczak W, et al. Acalabrutinib with or without obinutuzumab versus chlorambucil and obinutuzumab for treatment-naive chronic lymphocytic leukaemia (ELEVATE $\mathrm{TN})$ : a randomised, controlled, phase 3 trial. Lancet. 2020;395(10232):1278-91.

16. Vreman RA, Geenen JW, Hovels AM, Goettsch WG, Leufkens HGM, Al MJ. Phase I/II clinical trial-based early economic evaluation of acalabrutinib for relapsed chronic lymphocytic leukaemia. Appl Health Econ Health Policy. 2019;17(6):883-93.

17. Imbruvica (ibrutinib). Prescribing information. Janssen Biotech Inc; 2020. Accessed September 22, 2020. https:// www.imbruvica.com/files/prescribinginformation.pdf

18. National Cancer Institute. Combination therapy with venetoclax approved for chronic lymphocytic leukemia. June 17, 2019. Accessed 28 December 2020. https://www.cancer.gov/news-events/ cancer-currents-blog/2019/venetoclaxobinutuzumab-fda-approval-cll-sll

19. Al-Sawaf O, Zhang C, Tandon M, et al. Venetoclax plus obinutuzumab versus chlorambucil plus obinutuzumab for previously untreated chronic lymphocytic leukaemia (CLL14): follow-up results from a multicentre, open-label, randomised, phase 3 trial. Lancet Oncol. 2020;21(9): $1188-200$
20. Liu D, Zhao J. Frontline therapies for untreated chronic lymphoid leukemia. Exp Hematol Oncol. 2019;8:15.

21. Cho SK, Manzoor BS, Sail KR, et al. Budget impact of 12-month fixed treatment duration venetoclax in combination with obinutuzumab in previously untreated chronic lymphocytic leukemia patients in the United States. Pharmacoeconomics. 2020;38(9):941-51.

22. Weinstein MC, O'Brien B, Hornberger J, et al. Principles of good practice for decision analytic modeling in health-care evaluation: report of the ISPOR Task Force on Good Research Practices-Modeling Studies. Value Health. 2003;6(1):9-17.

23. National Institute for Health and Care Excellence (NICE). Guide to the methods of technology appraisal 2013. April 4, 2013. Accessed November 23, 2020. https://www.nice.org. uk/process/pmg9/resources/ guide-to-the-methods-of-technologyappraisal-2013-pdf-2007975843781

24. National Institute for Health and Care Excellence (NICE). Venetoclax with obinutuzumab for untreated chronic lymphocytic leukaemia. Technology appraisal guidance (TA663). December 9, 2020. Accessed December 22, 2020. https:// www.nice.org.uk/guidance/TA663

25. Ara R, Wailoo A. NICE DSU Technical Support Document 12: The Use of Health State Utility Values in Decision Models. National Institute for Health and Care Excellence (NICE); 2011.

26. Du Bois D, Du Bois EF. Clinical calorimetry: tenth paper a formula to estimate the approximate surface area if height and weight be known. Arch Intern Med (Chic). 1916;XVII(6_2):863-71.

27. Latimer N. NICE DSU technical support document 14: Survival analysis for economic evaluations alongside clinical trials - extrapolation with patient-level data. Updated March 2013. Accessed June 9, 2021. http://nicedsu. org.uk/wp-content/uploads/2016/03/ NICE-DSU-TSD-Survival-analysis. updated-March-2013.v2.pdf 
28. Arias E, Xu J. United States life tables, 2017. Natl Vital Stat Rep. 2019;68(7):1-66. Accessed June 9, 2021. https://www.cdc. gov/nchs/data/nvsr/nvsr68/nvsr68_07508.pdf

29. Sudhapalli P, Piena M, Palaka A, et al. Systematic literature review and network meta-analysis comparing therapies for treatment-naive patients with chronic lymphocytic leukemia. Eposter presented at: European Hematology Association, June 2020. Accessed June 9, 2021. https://library.ehaweb.org/eha/2020/ eha25th/294643/kavita.sail.systematic. literature.review.and.network.meta-analysis.comparing.html

30. Barr PM, Robak T, Owen C, et al. Sustained efficacy and detailed clinical follow-up of first-line ibrutinib treatment in older patients with chronic lymphocytic leukemia: extended phase 3 results from RESONATE-2. Haematologica. 2018;103(9):1502-10.

31. Moreno C, Greil R, Demirkan F, et al. Ibrutinib plus obinutuzumab versus chlorambucil plus obinutuzumab in firstline treatment of chronic lymphocytic leukaemia (iLLUMINATE): a multicentre, randomised, open-label, phase 3 trial. Lancet Oncol. 2019;20(1):43-56.

32. Woyach JA, Ruppert AS, Heerema NA, et al. Ibrutinib regimens versus chemoimmunotherapy in older patients with untreated CLL. N Engl J Med. 2018;379(26):2517-28.

33. National Institute for Health and Care Excellence (NICE). Obinutuzumab in combination with chlorambucil for untreated chronic lymphocytic leukaemia. Technology appraisal guidance [TA343]. June 2, 2015. Accessed September 22, 2020. https://www.nice.org.uk/guidance/ $\underline{\text { ta343 }}$

34. National Institute for Health and Care Excellence (NICE). Pixantrone monotherapy for treating multiply relapsed or refractory aggressive non-Hodgkin's B-cell lymphoma. Technology appraisal guidance [TA306]. February 26, 2014. Accessed September 22, 2020. https:// www.nice.org.uk/guidance/ta306
35. National Institute for Health and Care Excellence (NICE). Bendamustine for the first-line treatment of chronic lymphocytic leukaemia. Technology appraisal guidance [TA216]. February 23, 2011. Accessed September 22, 2020. https:// www.nice.org.uk/guidance/ta216

36. Nafees B, Stafford M, Gavriel S, Bhalla S, Watkins J. Health state utilities for non small cell lung cancer. Health Qual Life Outcomes. 2008;6:84.

37. Tolley K, Goad C, Yi Y, Maroudas P, Haiderali A, Thompson G. Utility elicitation study in the UK general public for late-stage chronic lymphocytic leukaemia. Eur J Health Econ. 2013;14(5):749-59.

38. National Institute for Health and Care Excellence (NICE). Idelalisib for treating chronic lymphocytic leukaemia. Technology appraisal guidance [TA359]. October 28, 2015. Accessed September 22, 2020. https://www.nice.org.uk/guidance/ ta359

39. Beusterien KM, Davies J, Leach M, et al. Population preference values for treatment outcomes in chronic lymphocytic leukaemia: a cross-sectional utility study. Health Qual Life Outcomes. 2010;8:50.

40. Lloyd A, Nafees B, Narewska J, Dewilde S, Watkins J. Health state utilities for metastatic breast cancer. $\mathrm{Br} \mathrm{J}$ Cancer. 2006;95(6):683-90.

41. Kater AP, Seymour JF, Hillmen P, et al. Fixed duration of venetoclax-rituximab in relapsed/refractory chronic lymphocytic leukemia eradicates minimal residual disease and prolongs survival: post-treatment follow-up of the MURANO Phase III study. J Clin Oncol. 2019;37(4):269-77.

42. O'Brien S, Furman RR, Coutre S, et al. Single-agent ibrutinib in treatment-naive and relapsed/refractory chronic lymphocytic leukemia: a 5-year experience. Blood. 2018;131(17):1910-19.

43. Davids MS, Hallek M, Wierda W, et al. Comprehensive safety analysis of venetoclax monotherapy for patients with relapsed/refractory chronic lymphocytic leukemia. Clin Cancer Res. 2018;24(18):4371-79.
44. Borker R. Costs associated with adverse events in patients with metastatic renal cell carcinoma. J Med Econ. 2014;17(11):792-97.

45. Barnes JI, Divi V, Begaye A, et al. Cost-effectiveness of ibrutinib as first-line therapy for chronic lymphocytic leukemia in older adults without deletion 17p. Blood Adv. 2018;2(15):1946-56.

46. Healthcare Cost and Utilization Project. National Inpatient Sample. Database. 2016. Agency for Healthcare Research and Quality. Accessed June 9, 2021. https://www.hcup-us.ahrq.gov/ nisoverview.jsp.

47. Wong W, Yim YM, Kim A, et al. Assessment of costs associated with adverse events in patients with cancer. PLoS One. 2018;13(4):e0196007.

48. Lafeuille MH, Vekeman F, Wang ST, Kerrigan M, Menditto L, Duh MS. Lifetime costs to Medicare of providing care to patients with chronic lymphocytic leukemia. Leuk Lymphoma. 2012;53(6):1146-54.

49. US Bureau of Labor Statistics. Databases, tables \& calculators by subject. 2020. Accessed November 26, 2020. https://data.bls.gov/timeseries/ CUUR0000SAM?output_view=data

50. Wang S, Lafeuille MH, Lefebvre P, Romdhani H, Emond B, Senbetta M. Economic burden of treatment failure in chronic lymphocytic leukemia patients. Curr Med Res Opin. 2018;34(6):1135-42.

51. Neumann PJ, Cohen JT, Weinstein MC. Updating cost-effectiveness-the curious resilience of the $\$ 50,000-$ perQALY threshold. N Engl J Med. 2014;371(9):796-97.

52. Hartung DM. Economics and costeffectiveness of multiple sclerosis therapies in the USA. Neurotherapeutics. 2017;14(4):1018-26.

53. Davids MS, Waweru C, Le Nouveau P, et al. Comparative efficacy of acalabrutinib in frontline treatment of chronic lymphocytic leukemia: a systematic review and network meta-analysis. Clin Ther. 2020;42(10):1955-74.e15. 
54. Sheng Z, Song S, Yu M, et al. Comparison of acalabrutinib plus obinutuzumab, ibrutinib plus obinutuzumab and venetoclax plus obinutuzumab for untreated CLL: a network meta-analysis. Leuk Lymphoma. 2020;61(14):3432-39.

55. Fischer K, Cramer P, Busch R, et al. Bendamustine in combination with rituximab for previously untreated patients with chronic lymphocytic leukemia: a multicenter phase II trial of the German Chronic Lymphocytic Leukemia Study Group. J Clin Oncol. 2012;30(26):3209-16.
56. ClinicalTrials.gov. A study of MabThera added to bendamustine or chlorambucil in patients with chronic lymphocytic leukemia (MaBLe). Identifier: NCT01056510. 2015. Accessed June 9, 2021. https://clinicaltrials.gov/ct2/show/NCT01056510

57. Fort N, Agathou S. Ibrutinib plus rituximab provide superior outcomes than FCR in naïve CLL patients: results from the ECOG-ACRIN phase III trial E1912. LymphomaHub. January 29, 2019. Accessed June 9, 2021. https://lymphomahub.com/medical-information/ ash-2018-ibrutinib-plus-rituximabprovide-superior-outcomes-than-fcr-innaive-cll-patients-results-from-the-ecogacrin-phase-iii-trial-e1912 